\title{
THE CONCEPT AND DESIGN OF PLEATED PNEUMATIC ARTIFICIAL MUSCLES
}

\author{
Frank Daerden, Dirk Lefeber
}

Vrije Universiteit Brussel, Dept. of Mechanical Engineering, Multibody Mechanics Research Group, Pleinlaan 2, 1050 Brussel, Belgiumfrank.daerden@vub.ac.be

\begin{abstract}
This paper describes the design of a new type of Pneumatic Artificial Muscle (PAM), namely the Pleated Pneumatic Artificial Muscle (PPAM). It was developed as an improvement with regard to existing types of PAM, e.g. the McKibben muscle. Its principle characteristic is its pleated membrane. It can inflate without material stretching and friction and has practically no stress in the direction perpendicular to its axis of symmetry. Besides these it is extremely strong and yet very lightweight and it has a large stroke compared to other designs. A general introduction on PAMs is given together with a short discussion and motivation for this new design. The concept of the PPAM is explained and a mathematical model is derived. This model proves its principle of operation. From the model, several characteristics, such as developed force, maximum contraction, diameter, volume and membrane tensile stress, are obtained. Material choices and dimensions of a typical PPAM are next discussed and its measured values of static force and diameter are compared to the model predicted values. The agreement between both is found to be very good.
\end{abstract}

Keywords: $\quad$ pleated pneumatic artificial muscle, PAM, PPAM, McKibben muscle, pneumatic, actuator, lightweight, compliance.

\section{Introduction}

Pneumatic Artificial Muscles (PAMs) are contractile and linear motion engines operated by gas pressure. Their concept is very simple: the actuator's core element is a flexible reinforced closed membrane attached at both ends to fittings along which mechanical power is transferred to a load. As the membrane is inflated or gas is sucked out of it, it bulges outward or is squeezed, respectively. Together with this radial expansion or contraction, the shell contracts axially and thereby exerts a pulling force on its load. The force and motion thus generated by this type of actuator are linear and unidirectional. This contractile operation distinguishes the PAM from bellows, which extend upon inflation. Although it is possible to design an underpressure operating muscle, e.g. Morin (1953) and Marcinčin and Palko (1993), PAMs usually operate at an overpressure since a lot more energy can be conveyed that way.

Assuming quasi-static conditions and neglecting energy losses and the energy needed to deform the membrane, the generated force can be put as

$$
F=-p \frac{d V}{d l_{c}}
$$

where $p$ is the pressure difference with regard to the surroundings (gauge pressure), $V$ the enclosed membrane volume and $l_{c}$ the muscle contracted length, cf. Fig. 1. In reality however, energy losses and deformation energy cannot usually be disregarded and the developed force will have a lower value. Nevertheless, Eq. 1 shows how the generated force depends on the applied gauge pressure and on the change of volume with regard to length - which is characteristic of the type of membrane used. The state of a particular kind of PAM is thus fully determined by its length and gauge pressure.

PAMs are extremely lightweight actuators because basically they are no more than a membrane. Power to weight ratios in excess of $1 \mathrm{~kW} / \mathrm{kg}$ are reported, see Caldwell et al. (1995), Hannaford et al. (1995) and Daerden (1999). Besides this, PAMs are characterised by their compliance which is due not only to gas compressibility but also to the varying force to displacement. Therefore, compliance can easily be adjusted and controlled, Daerden et al. (1999). These actuators can also be directly connected to a robotic joint because of their high output forces at all speeds. No gears have to be used and therefore no inertia or backlash is introduced. Moreover, PAMs are easy to connect and do not bring about hazards like fire, explosion, electric shock 
or pollution.

Ever since their first conceiving, which the authors could trace back to a patent by Sensaud de Lavaud (1929), fluid-driven muscle-like actuators of a number of kinds have been developed. Most of these were either too complex or too unreliable to be practicable, e.g. the designs by Baldwin, Kukolj, Yarlott, Paynter, Marcinčin and Kleinwachter-see Daerden (1999). Braided muscles, also called McKibben muscles, are most commonly used to date. This is probably because of their simple design and assembly: they are composed of a gas-tight elastic tube or bladder surrounded by a double helically braided sleeve. When pressurised the tube presses laterally against the sleeve. By changing its braid angle the braid changes its length, diameter and volume. According to Baldwin (1969), J. L. McKibben introduced this type of PAM as an orthotic actuator in the late 1950's: due to the similarity in length-load curves between this artificial muscle and skeletal muscle, it seemed an ideal choice for this purpose, as stated by Schulte (1961). However, practical problems such as pneumatic power storage or availability and poor quality valve technology at that time, gradually reduced interest from the prosthesis/orthotics community in McKibben's muscles. In the late 1980's it was reintroduced by Bridgestone Co. in Japan as the Rubbertuator, Inoue (1987), and used to power an industrial use robot arm, Soft Arm. Recently, a variant on the McKibben design by the name of MAS was designed and marketed by Festo AG. Several research groups use PAMs to power robots-mainly of anthropomorphic design - and prostheses and orthotics, e.g. Greenhill (1993), Hesselroth et al. (1994), Hannaford et al. (1995), Winters (1995), Noritsugu and Tanaka (1997), Tondu (1997), Caldwell et al. (1998) and Verrelst et al. (2000).

In spite of its widespread use, the braided muscle has important drawbacks as will be seen further. This paper presents a new design of PAM by Daerden (1999), namely the Pleated PAM (PPAM). It will be shown that this designs is an improvement compared to the braided muscle with regard to several actuator characteristics.

\section{Pleated PAM}

\subsection{Goal}

The Pleated PAM was developed because of several weak points of the braided muscles. Firstly, due to dry friction between the braid and the tube these actuators have substantial hysteresis, see Chou and Hannaford (1996) and Tondu et al. (1995). This has an adverse effect on actuator behaviour and necessitates the use of complex actuator models and control, e.g. Tondu (1997) and Caldwell et al. (1995). Secondly, deformation of the rubber tube lowers the generated force because of the energy it requires. This effect depends off course on the toughness of the rubber used, the tougher the rubber, the stronger the effect. Thirdly, the applied pressure has to exceed a threshold value to start the expansion of the tube. This value also depends on the toughness of the rubber, a value of about $100 \mathrm{kPa}$ for typical McKibben Muscles is cited by Chou and Hannaford (1996). A fourth weak point, also related to the membrane material, is failure. Klute and Hannaford (1998) describe rubber fatigue failure as the most common failure mode. Many users also complain of wires snapping at the end point of the actuator, i.e. where the tube and netting are clamped together. In addition to the above cited drawbacks, the displacement capability of braided muscles is rather limited. Total displacement or stroke, which is usually expressed as a percentage with regard to a reference length, ranges between $20 \%$ to $30 \%$ of the length at rest, see Chou and Hannaford (1996), Inoue (1987) and Tondu et al. (1995).

Other types of PAM have some improvements with regard to these points but often introduce other disadvantages. ROMAC muscles for instance, Immega (1986), can generate very high forces and are not bothered by friction and material deformation. Contraction can be up to $50 \%$ of the initial length. Their major drawback, however, is their complex shape, making them difficult to construct. On top of this, ROMACs are netted muscles, i.e. they consist of a membrane encompassed by a netting, and are therefore characterised by strong stress concentrations in the surrounding wires and, hence, early material failure.

Thus, the improved PAM design that was looked for had to show: (1) low membrane material deformation, (2) no, or at least very low friction/hysteresis, (3) high tensile strength in the longitudinal direction, (4) uniform membrane loading, (5) low threshold pressure, (6) high maximum pressure and (7) high maximum contraction.

\subsection{Concept}

From the discussion above, it is clear that material deformation should be avoided. This can be done by using membrane rearranging in order to allow for inflation. The principle of rearranging is to have a membrane that in some way unfurls as it is inflated. When such PAMs contract their membranes' surface area do not change contrary to the increasing surface area of deforming membranes.

The basic idea of the PPAM was to do this by using a cylindrical membrane of a high tensile stiffness and high flexibility and folding it together along its central axis, like accordion bellows. The membrane material needed to expand is thus stashed away inside the folds. At both ends the membrane is tightly locked to fittings, which also carry the gas inlet and outlet ducts. When such a PAM is pressurised it shortens and bulges. As the membrane has a high tensile stiffness, the expansion is highest in the middle of the membrane and gradually goes down toward both ends where no expansion at all can occur. Inflation therefore happens by unfolding, as is shown in Fig. 1. Because the fold faces are laid out radially no friction is involved in this process and no friction related hysteresis will occur. Furthermore, unfolding needs no appreciable amount of energy so no loss of output force will ensue from this. 
(a)

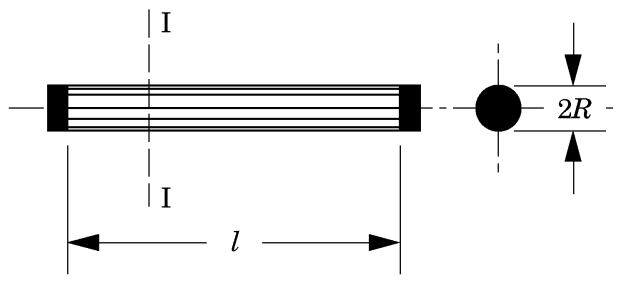

(b)

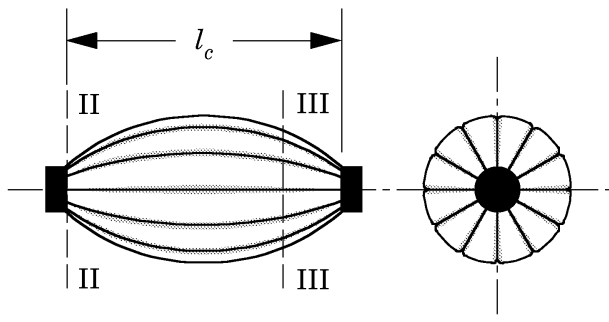

I-I:

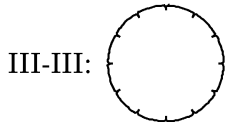

Fig. 1: Pleated PAM, (a) uninflated, at rest and (b) inflated.

Ideally, such a PAM would have an infinite amount of infinitely narrow pleats, leading to an axisymmetrical membrane surface that would thus only be loaded by meridional stresses (i.e. along fold lines) and not by parallel stresses (i.e. along parallels, which are sections of the surface and any plane perpendicular to the axis of symmetry). This can be seen as follows. If any parallel stress would exist in such a membrane at some equilibrium contraction, it would unfold the membrane further, since folds cannot withstand tensile stress. As a result of this, the membrane diameter would have to increase, which, at a fixed contraction, can only happen by stretching in the meridional direction. The high tensile stiffness of the material makes it nearly unstretchable though and, therefore, unfolding can only happen if the membrane contracts further. An ideal PPAM, consequently, cannot have parallel stress components. Its lateral expansion can happen unhampered and at no energy cost.

\subsection{Mathematical model}

The ideal pleated PAM can mathematically be described by an orthotropic membrane that is closed, flexible, axisymmetrical and subjected to an axial force $F$ at both ends and to a uniform orthogonal surface load $p$, which is the pressure difference across the surface. This orthotropic membrane has a zero modulus of Young in the parallel direction but not in the meridional direction. This amounts to a zero parallel stress condition and actually states that the external loads are balanced by meridional membrane stress only. As inflation is done by unfurling the infinitely small folds the membrane parallel cross section area, i.e. sheet thickness times circumference, never changes. It has the same value at any parallel section and is unaffected by the state of inflation.

The surface of this membrane is generated by a meridian curve that is revolved about the axis of symmetry, the $x$-axis. From a static force equilibrium analysis of the membrane, a relation between the shape of the generating curve, the membrane meridional tensile stress $\sigma$, the applied gauge pressure $p$ and the developed force $F$ can be established, see Daerden (1999). Because of axisymmetry, two force equilibrium equations are obtained:

$$
\begin{aligned}
& \pi p r^{2}+F=\frac{2 \pi \sigma s r}{\sqrt{1+r^{\prime 2}}} \\
& (\sigma s r)^{\prime}=0
\end{aligned}
$$

where $r$ represents the meridian's distance to the $x$ axis, $s$ the sheet thickness and the derivative is with respect to $x$.

In order to solve it, it is completed by two equations expressing linear elastic material behaviour in the meridional direction and the constant parallel membrane cross section area $A$ :

$$
\begin{aligned}
& 2 \int_{0}^{l_{c} / 2} \frac{\sqrt{1+r^{\prime 2}}}{1+\frac{\sigma}{E}} d x=l \\
& 2 \pi r s=A
\end{aligned}
$$

with $x=0$ at the membrane centre. The boundary conditions for this set are $r\left(l_{c} / 2\right)=R$, which is the membrane uninflated radius, cf. Fig. 1 , and $r^{\prime}(0)=0$. For every possible value of contraction, which is defined as

$$
\varepsilon=1-\frac{l_{c}}{l}
$$

with $l_{c}$ and $l$ as in Fig. 1, the set of Eqs. 2 to 5 of the unknowns $r, \sigma, F$ and $s$ has to be solved. This involves the use of elliptic integrals of the first and second kind, which are defined as

$$
\begin{aligned}
& \mathrm{F}(\varphi \backslash m)=\int_{0}^{\varphi} \frac{d \theta}{\sqrt{1-m \sin ^{2} \theta}} \\
& \mathrm{E}(\varphi \backslash m)=\int_{0}^{\varphi} \frac{d \theta}{\sqrt{1-m \sin ^{2} \theta}}
\end{aligned}
$$

respectively.

\subsection{Characteristics}

The meridian curve or $r(x)$ is given by a parametric representation $x(\varphi)-r(\varphi)$ :

$$
\begin{aligned}
& x=\frac{R}{\sqrt{m} \cos \varphi_{R}}\left(\mathrm{E}(\varphi \backslash m)-\frac{1}{2} \mathrm{~F}(\varphi \backslash m)\right) \\
& r=\frac{R}{\cos \varphi_{R}} \cos \varphi
\end{aligned}
$$

where $\varphi$ runs from zero to some value $\varphi_{R}$. The constants $m$ and $\varphi_{R}$ are determined from

$$
\begin{aligned}
& \frac{2 \mathrm{E}\left(\varphi_{R} \backslash m\right)-\mathrm{F}\left(\varphi_{R} \backslash m\right)}{\sqrt{m} \cos \varphi_{R}}=\frac{l}{R}(1-\varepsilon) \\
& \frac{\mathrm{F}\left(\varphi_{R} \backslash m\right)}{\sqrt{m} \cos \varphi_{R}}=\frac{l}{R}\left(1+\frac{a}{2 m \cos ^{2} \varphi_{R}}\right)
\end{aligned}
$$


In the latter equation $a$ takes into account the elastic behaviour in the meridional direction: $a=\pi p R^{2} / A E$, with $E$ the modulus of Young.

Figure 2 shows the meridians for a membrane of $l=10 \mathrm{~cm}, \quad R=1 \mathrm{~cm}, \quad$ and $a=10^{-4} \quad\left(A=31.4 \mathrm{~mm}^{2}\right.$, $E=10 \mathrm{GPa}, \quad p=100 \mathrm{kPa}$ ) for various values of contraction. As can be seen from the diagram, even at a negative contraction of the membrane, i.e. elongation, it bulges slightly outward due to elasticity. This effect is all the less as the membrane's tensile stiffness in the meridional direction increases.

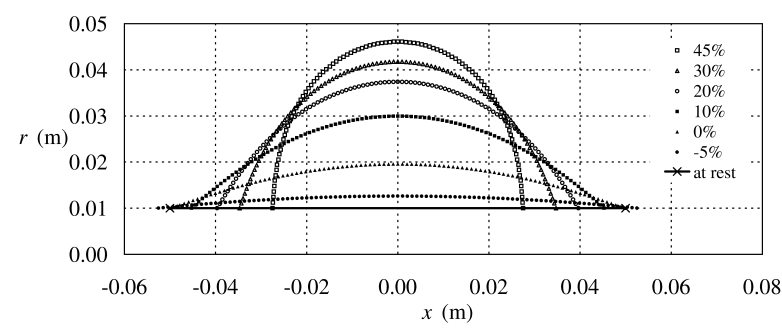

Fig 2: Meridians of a zero parallel stress membrane at various contractions.

All other characteristics are expressed as the product of a dimensionless function and a factor of the appropriate dimensions. These dimensionless functions are determined by $m$ and $\varphi_{R}$ and thus only depend on contraction, on $l / R$, which is called the muscle slenderness, and on $a$ as can be seen from Eqs. 11 and 12 . As an example the developed pulling force is found to be

$$
F=p l^{2} f\left(\varepsilon, \frac{l}{R}, a\right)
$$

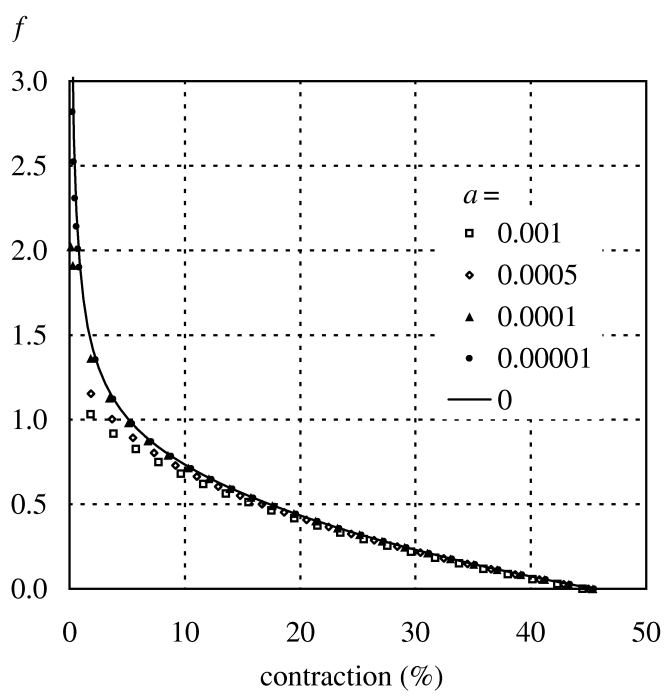

Fig. 3: Dimensionless force function.

The dimensionless force function $f$ is diagrammed in Fig. 3 for a slenderness of 10 to 1 . This shows the actuator force to be a non-linear function of displacement. At full length or zero contraction the developed force is extremely high but as contraction goes on it drops gradually toward zero. At that point the actuator has reached its maximum contraction. The influence of the membrane's elasticity can be seen from the diagram. At low contractions the developed force is considerably lowered because of bulging due to membrane strain. This means that, in practice, the developed force will never be infinitely high as would be the case for an inelastic membrane ( $E=\infty$ or $a=0$ ). As contraction grows beyond $5 \%$ the influence is only moderate. The maximum contraction is somewhat lowered, which is less discernible on the diagram: stroke is $45.5 \%$ for an inelastic membrane and $44.5 \%$ when $a=0.001$.

Because the effect of elasticity is very small at contractions of more than $5 \%$ for a high tensile stiffness membrane, the value of $a$ can accordingly be set to zero and Eqs. 11 and 12 will then yield $m$ and $\varphi_{R}$ as a function of contraction and $l / R$ only. With this inelastic approximation, the following expressions were found for the pulling force, the meridional stress $\sigma$, the membrane central or equatorial diameter $D$ and its enclosed volume $V$ :

$$
\begin{aligned}
& F=p l^{2} f\left(\varepsilon, \frac{l}{R}\right) \\
& \sigma=\frac{p l^{2}}{A} \varsigma\left(\varepsilon, \frac{l}{R}\right) \\
& D=l d\left(\varepsilon, \frac{l}{R}\right) \\
& V=l^{3} v\left(\varepsilon, \frac{l}{R}\right)
\end{aligned}
$$

with $f, \varsigma, d$ and $v$ the according dimensionless functions. These expressions show how membranes of different sizes but identical slenderness have their characteristics scaled with size: central diameter with length, force with the square of length and volume with the cube of length. Meridional stress is independent of size if the sheet thickness is proportional to size. Force and stress are also proportional to the applied pressure level, as could be expected. If one intends to double the force at the same pressure while using geometrically similar membranes, length has to increase by only $41 \%$, but volume-and thus pressurised gas consumptionwill then increase by $183 \%$. However this also implies contraction lengths being $41 \%$ larger, which is not always needed. On the other hand, if one does not need high forces it is possible to replace one long membrane by a series of $n$ similar but shorter ones so that total length and contraction are preserved but total volume and force are divided by $n^{2}$.

Figure 4 shows the inelastic approximation of $f$ as a function of contraction for various values of membrane slenderness. Except for the hypothetical case of an infinitely thin muscle force will tend to infinity for zero contraction. In reality however, due to elasticity the force levels will drop at low contractions, which could already be seen from Fig. 3. Stubby membranes, i.e. low value of slenderness, are clearly stronger at low contractions but their developed force also drops at a steeper pace. This is because in stubby membranes the gas has a much greater surface against which to act but at the same time the end closure surfaces and thereby the pressure load acting against them increase. 


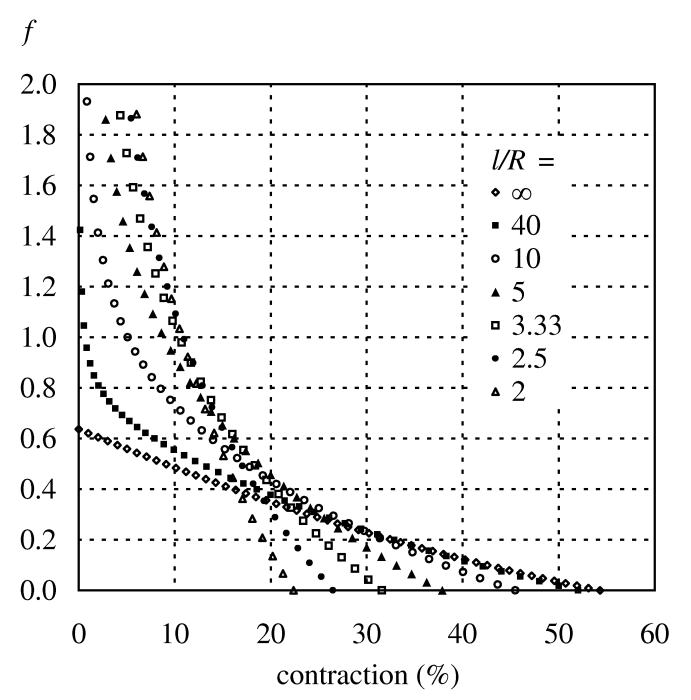

Fig. 4: Dimensionless force function.

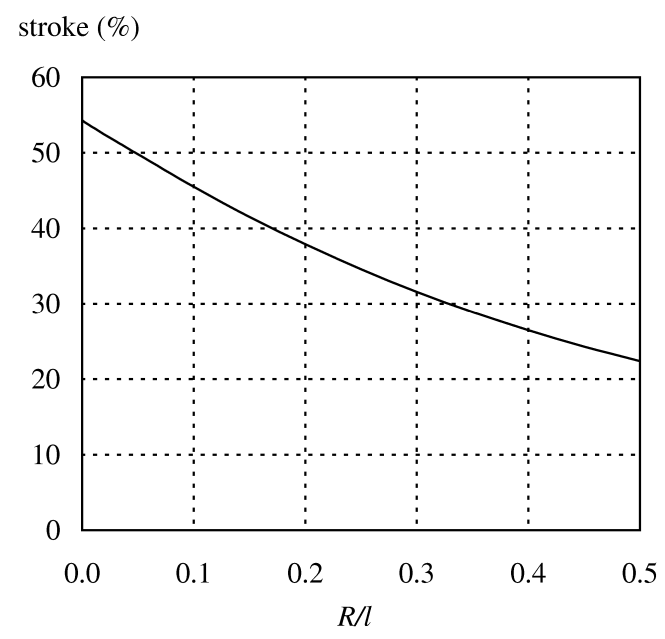

Fig. 5: Pleated PAM stroke.

Figure 5 plots the actuator stroke as function of the inverse of slenderness. Stroke is strongly dependent on slenderness: slim membranes have a greater stroke than stubbier ones. As $R$ increases with regard to $l$, the pressure load acting on the end closures increases and this will bring $F$ to zero at lower values of $\varepsilon$. An infinitely slim membrane has the greatest ability to contract with a stroke of about $54.3 \%$. This is a limiting case that will not be feasible in practice. At a more practicable slenderness of $l / R=10$ the maximum contraction is about $45.5 \%$, a membrane having an initial diameter equal to its length, has a total contraction of only $22.3 \%$.

The diagram of Fig. 6 sets the dimensionless function of meridional stress as a function of contraction. From Eqs. 3 and 5, it is clear that the meridional stress is the same throughout the membrane. The level of stress obviously never drops to zero: a fully inflated muscle does not produce any pulling force, but it is tense nonetheless.

Figure 7 shows how the central diameter evolves with contraction. It is clear how the relative expansion is larger for slimmer membranes. This implies that slimmer PPAMs actually need more folds to be able to expand fully and are, therefore, more difficult to build.
The value of $d$ at maximum contraction, $d^{\prime}$, is given in Fig. 8. This indicates how wide the muscle will get with regard to its full length, depending on its slenderness.

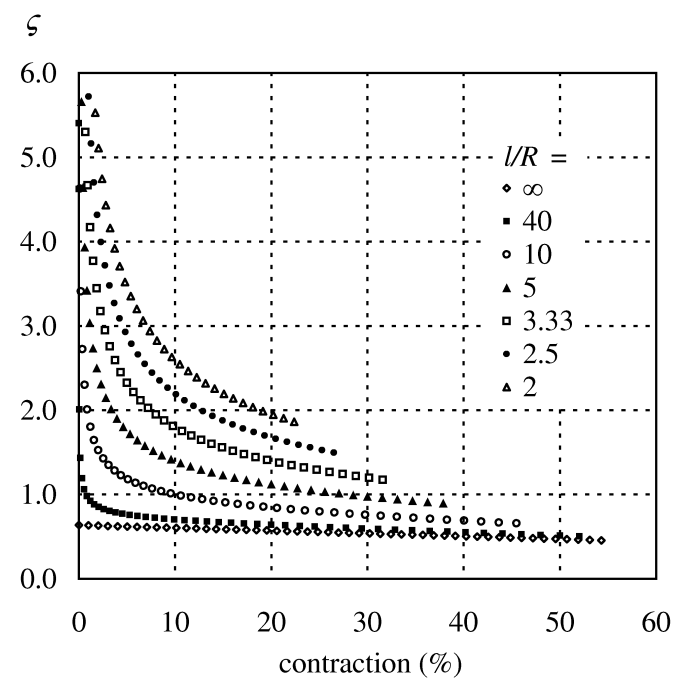

Fig. 6: Dimensionless stress function.

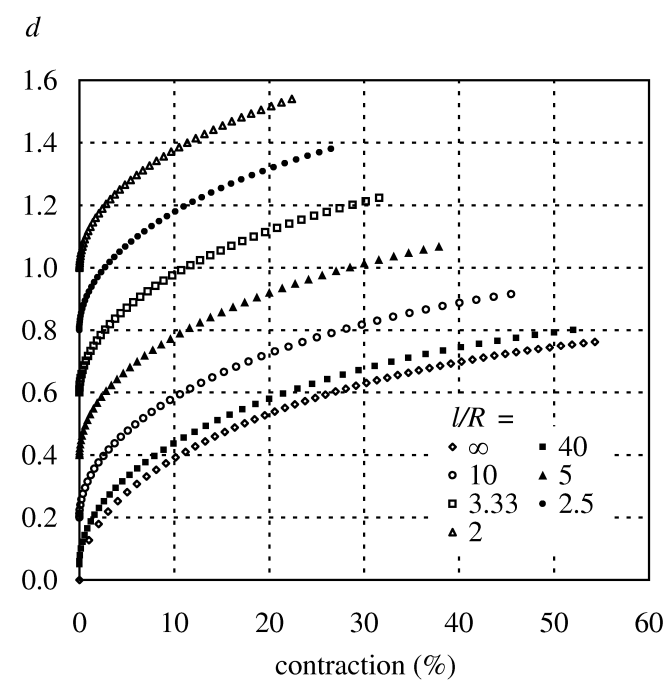

Fig. 7: Dimensionless diameter function.

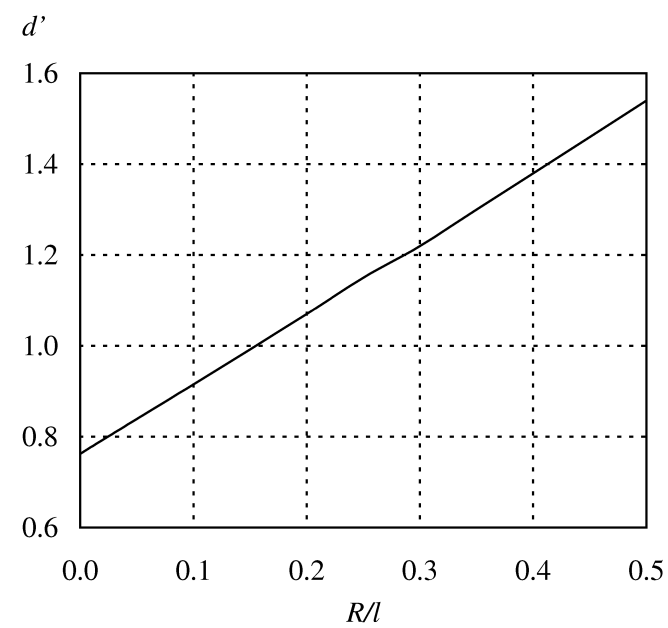

Fig. 8: Maximum values of diameter function. 
The evolution of volume with contraction is seen in Fig. 9. Although they bulge less, wider membranes have a higher increase in volume with contraction and, therefore, a higher possible transfer of work. Because of this and because they are easier to construct one could be inclined to favour stubby PPAMs. These have two important drawbacks, however. As they have a small travel and high force, they put higher loads to the structure they are connected to compared to slimmer ones transferring the same work. Secondly, they have a large dead volume (i.e. at $\varepsilon=0$ ), as can be seen from the diagram, and this has to be charged or discharged whenever changing the pressure inside the muscle although it does not contribute to the generation of force, cf. Eq. 1.

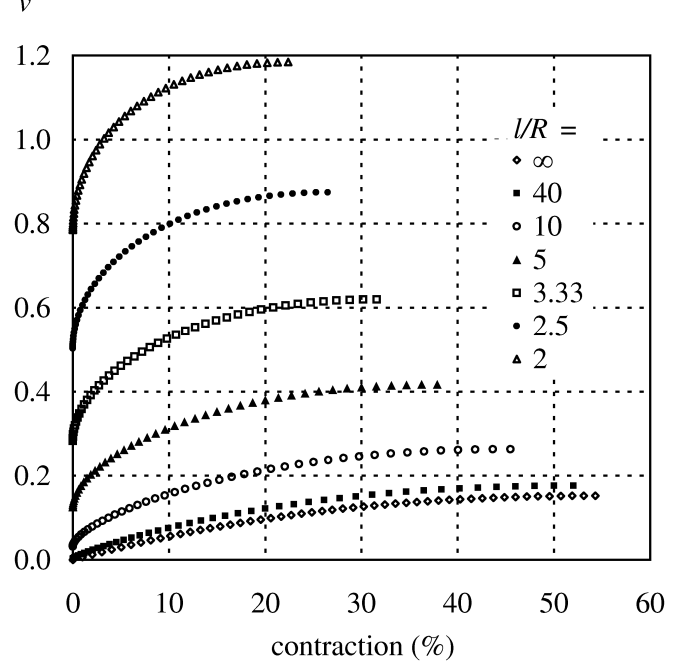

Fig. 9: Dimensionless volume function.

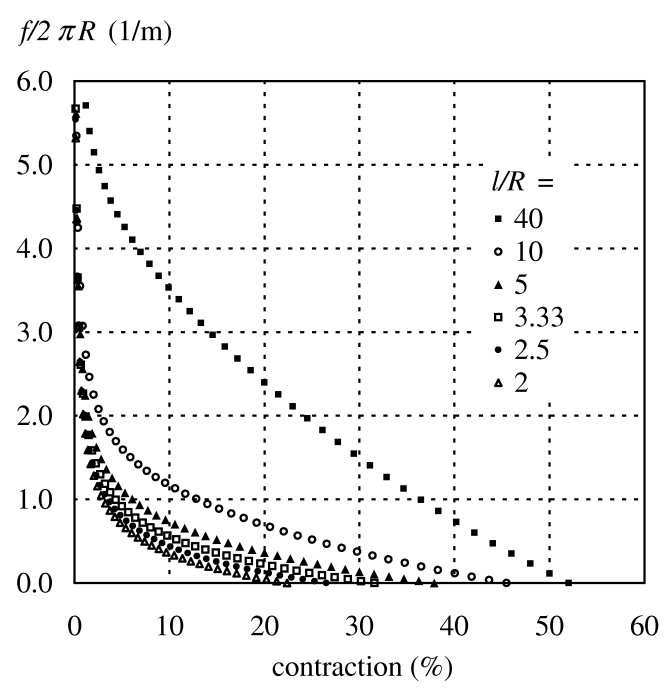

Fig. 10: Circumferential force concentration.

It is interesting to look at the plot of dimensionless force per unit length along the end fittings' circumference, or $f / 2 \pi R$, as shown in Fig. 10. Comparing actuators of the same length, the slimmer ones generate less pulling force but their force to circumference ratio increases. This implies that slender membranes impose higher stresses on the end fittings even though forces are generally lower. Together with the higher amount of folds needed, as stated above, this will put a lower limit on technically feasible values of slenderness.

\subsection{Goals check}

Going over the design goals that were set out in $\S 2.1$ it can now be seen that they can all be met by the concept of the pleated PAM and by an adequate choice of materials. (1) Material deformation can be kept to a minimum by choosing a high tensile stiffness material, e.g para-aramid with a Young's modulus over $100 \mathrm{GPa}$, and because of the expansion by membrane rearranging. (2) Friction and, hence, hysteresis are nearly eliminated by the principle of folding/unfolding and because no outer braid or netting is used. (3) High tensile strength in the longitudinal direction is obtained by choosing the right material. (4) The stress distribution is uniform for an ideal PPAM, as was seen above. Because of the limited amount of pleats non-uniformities will be present though in real pleated PAMs. These can be expected to grow as the amount of pleats decreases, so putting in as much folds as possible will be important. (5) The threshold pressure will be determined by the flexibility of the membrane: pleating a sheet is known to increase its structural strength and resistance to deformation-in this case inflation-and this will be all the more so if the material is only moderately flexible. Values of $100 \mathrm{kPa}$, as is the case for some braided muscles, are very high compared to what can be expected for a pleated PAM, as will be seen in the next section. (6) The maximum allowable pressure will, in theory, be set by the tensile strength of the material. In practice, extra limiting factors will arise, e.g. stress concentration at the membrane to end fitting attachment. (7) The maximum contraction of this type of PAM depends on its slenderness: values of around $45 \%$ are easily obtained. Compared to other kinds of PAMs this is quite high a value.

\section{Practical realisation}

The surface of a pleated PAM is not axisymmetrical because of the limited amount of pleats. Instead, it has a rotationally repeated pattern of ridges and valleysprovided all pleats have the same dimensions. Therefore, parallel stress components and a meridional stress non-uniformity can be expected to exist. In order to keep these small and approximate the ideal membrane to a good degree, the number of folds ought to be as high as possible and their depth as small as possible.

As an example, this section describes a pleated muscle of $l=10 \mathrm{~cm}$ and $R=1.25 \mathrm{~cm}$. It has 44 folds, each having a depth of $2.5 \mathrm{~mm}$. With a pleat pitch of $7 \mathrm{~mm}$ the membrane circumference is $44 \times 7 \mathrm{~mm}$, allowing the membrane diameter to grow to $9.8 \mathrm{~cm}$. Calculations from the mathematical model presented above predict a maximum diameter of $9.54 \mathrm{~cm}$.

For the mechanical design, choices had to be made regarding the membrane and end fitting materials and regarding their connection method. A Kevlar ${ }^{\odot} 49$ fabric 
was chosen as primary membrane material. Kevlar ${ }^{\circledR} 49$, which is produced by DuPont de Nemours, is a paraaramid polymer. It is spun in filaments of $12 \mu \mathrm{m}$ diameter that are assembled in yarns in various quantities. From these yarns fabrics are woven. Kevlar ${ }^{\circledR} 49$ has a high tensile strength $(\approx 4 \mathrm{GPa})$ at a low specific weight $\left(1440 \mathrm{~kg} / \mathrm{m}^{3}\right)$, a high elastic modulus $(\approx 130 \mathrm{GPa})$, low creep and relaxation, high toughness, good fatigue resistance and non-brittle behaviour. The latter three characteristics are important for this application because of the possibly sharp bends at the fold lines, the end fittings and the points of membrane fixing. The fabric was sealed by a polypropylene lining which was glued to the fabric by a rubber resin adhesive. The resulting lining thickness is $0.08 \mathrm{~mm}$ and the total sheet thickness $0.28 \mathrm{~mm}$.

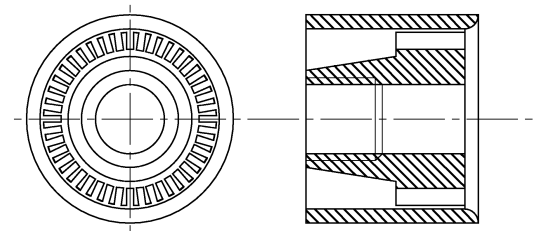

Fig. 11: Pleated PAM end fitting assembly.

Because of the extreme smoothness of the fibres a clamp was rejected as a means of fixing the membrane to the end fittings: in order to get a high enough friction force to fix the membrane the clamp pressure has to rise beyond the allowable lateral fibre pressure. A bonding method to secure the membrane was therefore used: inside the end fittings the fabric is embedded in an epoxy resin. The assembly of an end fitting is shown in Fig. 11 in a frontal view and a sideways axial cross section. It consists of a plug and an outer ring, both made of aluminium. The plug has a tapered side and a toothed side to it and is shifted into the ring. The membrane is inserted between both parts. The toothed side keeps the pleats into place and ensures them to be equidistant. Securing the fabric to the resin is done at the tapered side. No resin is allowed to enter the slots of the plug. As a result of this layout, there is only marginal tilting of the fibres at the spot where they come out of the resin. This is important because these are locations of stress concentration. The plug is hollow, which allows for external attachment of the load and inlet/outlet of pressurised air.

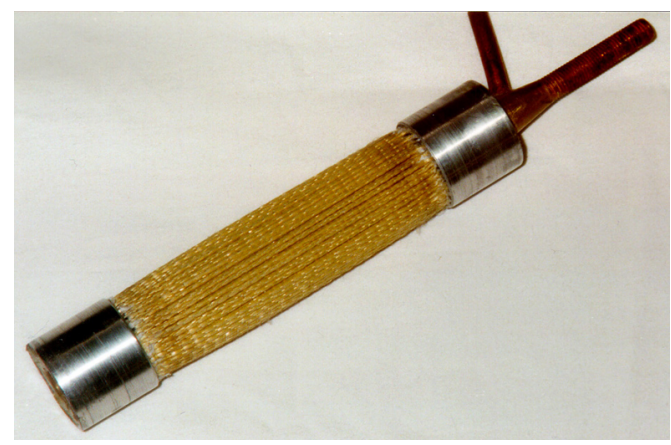

Fig. 12: Pleated PAM photograph.

The weights of the parts are $7.3 \mathrm{~g}$ for the membrane, $12.5 \mathrm{~g}$ for the plug, $1.8 \mathrm{~g}$ for the resin filling and
$11.2 \mathrm{~g}$ for the outer ring. The total muscle weight, using two basic plugs is thus $58.3 \mathrm{~g}$. A photograph of a prototype PPAM is shown in Fig. 12. One of its end fittings has an extended plug that has an air duct to one side.

\section{Measured characteristics}

The actuator described in the previous section was tested in a draw bench. The developed force and the equatorial diameter were recorded as a function of contraction for various values of gauge pressure. All test runs were repeated in order to determine the repeatability. The isobaric force and diameter diagrams were established and compared to those obtained from the mathematical model discussed in $\S 2.2$ and $\S 2.3$.

Figure 13 shows the measured force. In order to protect the actuator from excessive loading the force levels were limited to some $3500 \mathrm{~N}$ and gauge pressure to $300 \mathrm{kPa}$. Sustained exceeding of these levels led to gradual snapping of fibres at the interface of embedded and naked yarns, which are locations of stress concentrations, as was already mentioned above. Short pressure bursts of up to $700 \mathrm{kPa}$, however, did not harm the muscle. It was seen that scattering between test runs lay within $5 \%$ for pressures below $100 \mathrm{kPa}$. For higher pressures scattering was down to a few percents.

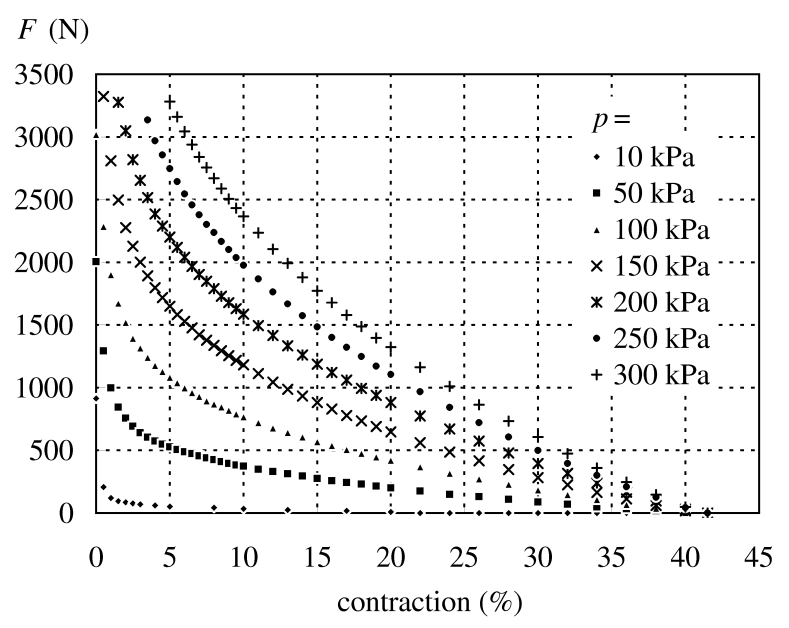

Fig. 13: Pleated PAM measured force.

The actuator stroke, which was predicted to be $43.5 \%$, was experimentally found to be $41.5 \%$. At this contraction, the equatorial section was not fully stretched or circular, indicating that further inflation is not hampered by lack of membrane circumference or shortage of folds. The deviation of $2 \%$ or $2 \mathrm{~mm}-$ similar values were found in all tested muscle models - is partly due to the membrane's elasticity, cf. Fig. 3, and partly to be attributed to the pleating. An exact determination of the mechanism responsible for this is, however, not possible from the model discussed in $\S 2.2$.

From the recorded force the dimensionless force function was determined for each gauge pressure. Figure 14 shows these measured dimensionless force functions. For low values of gauge pressure, i.e. below 
$50 \mathrm{kPa}$, the values of $f$ deviate from those at higher pressures. This is in disagreement with the mathematical model stating $f$ to be independent of pressure. The effect gradually decreases as the pressure increases. As from a gauge pressure of $50 \mathrm{kPa}$ the curves are nearly the same and as from $150 \mathrm{kPa}$ they actually coincide. The deviation is in fact the PPAM threshold pressure behaviour. As was explained before a pleated membrane is less flexible than the flat and unpleated piece of lined fabric it is made of. A few kPa's are sufficient to inflate and operate the PAM but not to fully stretch and bulge its surface. This can be visually observed: at low pressures the membrane yarns are clearly nonuniformly stressed, some regions even remain slack to some degree and this gives its surface a rough and dented appearance, at higher pressures, the membrane is more strongly forced outward, which makes it taut at every point and gives it a smooth and very tense appearance. It can be concluded that a single threshold pressure is difficult to distinct, a value of $10 \mathrm{kPa}$ could however be agreed upon as threshold value. The operating pressures thus range from $10 \mathrm{kPa}$ to $300 \mathrm{kPa}$ or over a ratio of 30 . For braided muscles this ratio is about 6 to 8 as can be concluded from Chou and Hannaford (1996).

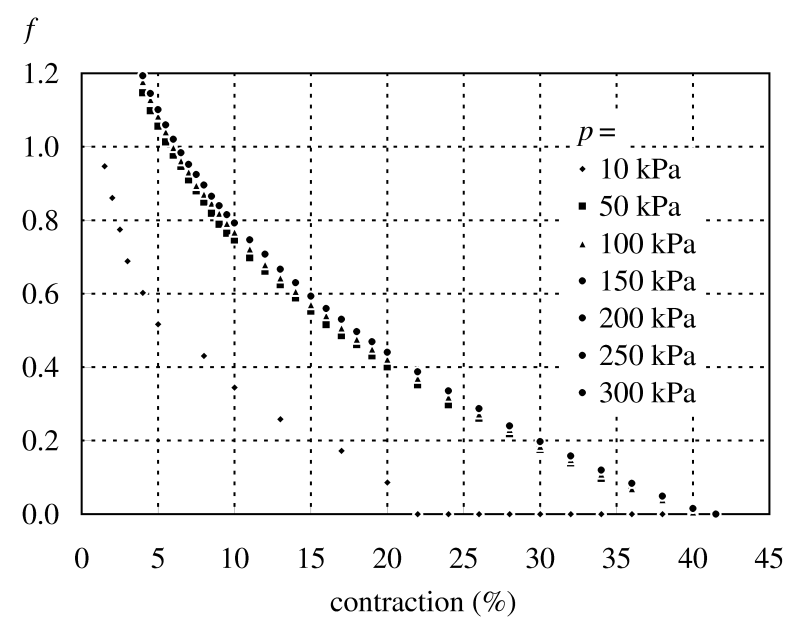

Fig. 14: Pleated Pam measured values of $f$.

Figure 15 plots the measured values of $f$ averaged for all tests at pressure levels above $100 \mathrm{kPa}$. Within the contraction range of $3 \%$ to $30 \%$ they match very well with the calculated values. At lower contractions, the membrane tensile compliance lowers the developed force to finite values, as was predicted by the model, cf. Fig. 3. At higher contractions, force drops to zero at a slightly steeper pace than predicted by the inelastic model. As explained above, this is partly due to the elastic behaviour and partly to the finite value of pleat depth.

Figure 16 diagrams the values of the measured and calculated dimensionless diameter function $d$. Only the values at gauge pressures of $50 \mathrm{kPa}$ and $300 \mathrm{kPa}$ are shown because the change in diameter is too small to be discernible on this scale. The agreement between calculated and measured values is very good. The effect of pressure is clearly noticeable at low contrac- tions, as was predicted by the zero parallel stress model. Except for very low pressures scattering between test runs was too low to be of any importance.

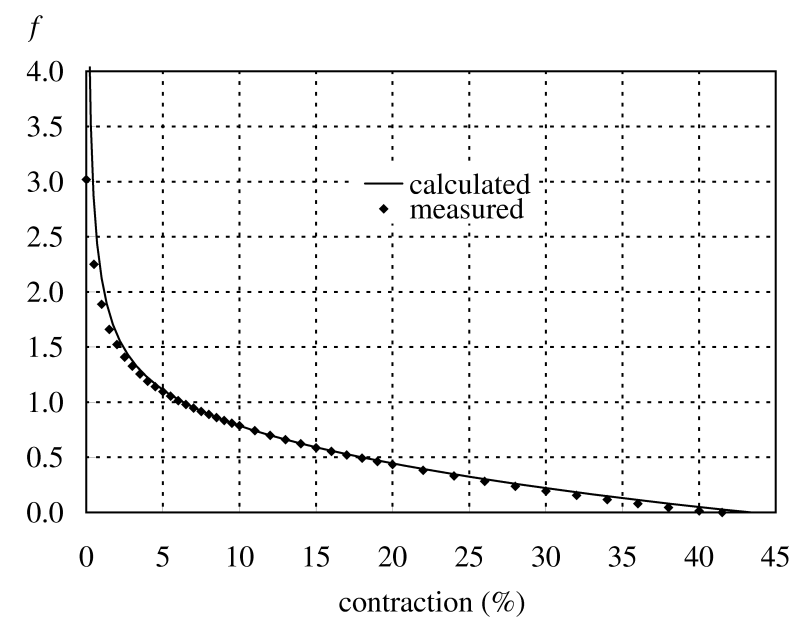

Fig. 15: Pleated Pam measured values of $f$, mean values.

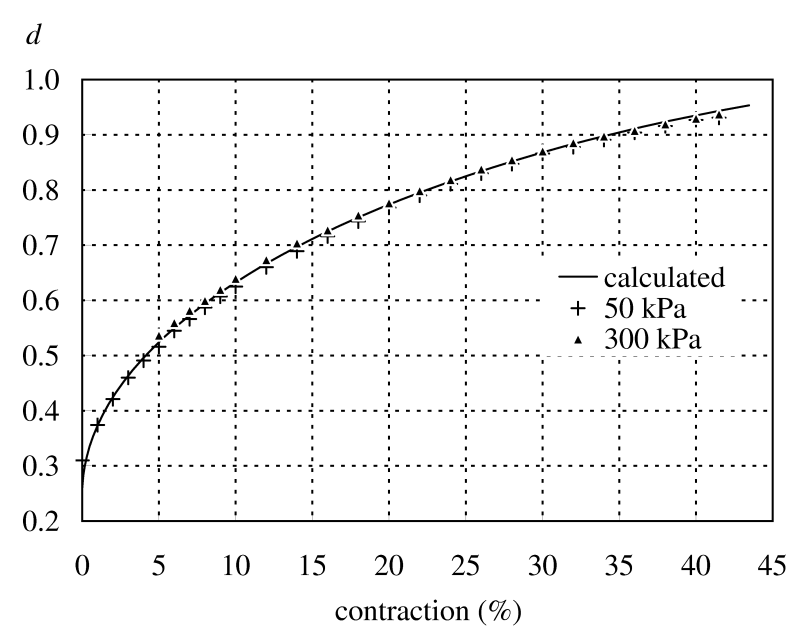

Fig. 16: Pleated PAM measured values of $d$.

These and other similar tests validated the mathematical model proposed in $\S 2.2$ for the pleated PAM. Furthermore, the inelastic model can effectively be used to describe the pleated actuator characteristics within the major part of its operating range of contraction. Slight deviations between inelastic model predicted and measured values do occur at extreme conditions, i.e. small and high contractions and low pressures, but these are not of great practical importance in normal operating conditions. In summary, the PPAM was found to be a reliable actuation device with a good degree of repeatability and predictability.

\section{Conclusions}

This paper described a new kind of pneumatic artificial muscle, namely the pleated PAM. The purpose of having a new design was explained together with its concept. A mathematical model was established and proved to be very accurate with regard to the real 
PPAM. The PPAM is very strong compared to other designs, has a larger stroke and is not bothered by friction related hysteresis. It is also extremely lightweight, a PPAM of less than $60 \mathrm{~g}$ was seen able to pull $3500 \mathrm{~N}$.

A PPAM is an ideal actuator whenever weight and compliance are important considerations. Examples of this are grippers with an adjustable firmness, strong but lightweight flexible robot arms and walking/running machines. Furthermore, PPAMS are easy to control, Daerden et al. (1999), and with them a very accurate positioning can be obtained, making them also suitable for this purpose. PPAMs can guarantee a safe manmachine interaction because of the delicate touch they can effect. This is important e.g. for handling fragile objects or to power rehabilitation devices, to name but two.

In summary, PPAMs have several characteristics that are not inherent to other, more classical drives, and are an improvement over existing PAMs. Therefore, the authors feel PPAMs are very suitable for a range of robotic and automation applications.

\section{References}

Baldwin, H. A. 1969. Realizable models of muscle function. Biomechanics, Proceedings of the First Rock Biomechanics Symposium, pp. 139-148, Plenum Press, New York.

Caldwell, D. G., Tsagarakis, N., Badihi, D. and Medrano-Cerda, G. A. 1998. Pneumatic muscle actuator technology: a lightweight power system for a humanoid robot. IEEE International Conference on Robotics and Automation, pp. 3035-3058, Leuven, Belgium.

Caldwell, D. G., Medrano-Cerda, G. A. and Goodwin, M. J. 1995. Control of pneumatic muscle actuators. IEEE Control Systems Magazine, Vol. 15(1), pp. 40-48.

Chou C.-P. and Hannaford, B. 1996. Measurement and modeling of McKibben pneumatic artificial muscles. IEEE Transactions on Robotics and Automation, Vol. 12(1), pp. 90-102.

Daerden, F. 1999. Conception and Realization of Pleated Pneumatic Artificial Muscles and their Use as Compliant Actuation Elements. PhD thesis. Vrije Universiteit Brussel, Belgium.

Daerden, F., Verrelst, B., Lefeber, D. and Kool, P. 1999. Controlling motion and compliance with folded pneumatic artificial muscles. Proceedings of CLAWAR '99: International Workshop and Conference, pp. 667-677, Portsmouth, UK.

Greenhill, S. 1993 The digit muscle. Industrial Robot, Vol. 20(5), pp. 29-30.

Hannaford, B., Winters, J. M., Chou, C.-P. and Marbot, P. H. 1995. The anthroform biorobotic arm: a system for the study of spinal circuits. In Annals of Biomedical Enineering, Vol. 23, pp. 399408 .
Hesselroth, T., Sarkar, K., van der Smaght, P. and Schulten, K. 1994. Neural network control of a pneumatic robot arm. IEEE Transactions on Systems, Man and Cybernatics, Vol. 24(1), pp. 2838.

Immega, G. B. 1986. ROMAC muscle powered robots. MS86-777, Society of Manufacturing Engineers, Dearborn.

Inoue, K. 1987. Rubbertuators and applications for robotics. 4th International Symposium on Robotics Research, pp. 57-63.

Klute, G. and Hannaford, B. 1998. Fatigue characteristics of McKibben artificial muscle actuators. Proceedings of IROS 1998, pp. 17761782, Victoria, B.C., Canada.

Marcinčin, J. and Palko, A. 1993. Negative pressure artificial muscle-An unconventional drive of robotic and handling systems. Transactions of the University of Košice, pp. 350-354, Riecansky Science Publishing Co, Slovak Republic.

Morin, A. H. 1953. Elastic Diaphragm. US Patent No. $2,642,091$.

Noritsugu, T. and Tanaka, T. 1997. Application of rubber artificial muscle manipulator as a rehabilitation robot. IEEE/ASME Transactions on Mechatronics, Vol. 2(4), pp. 259-267.

Schulte, H. F. 1961. The characteristics of the McKibben artificial muscle. The application of external power in prosthetics and orthotics, pp. 94115, Publication 874 of the National Academy of Sciences-National Research Council, Lake Arrowhead.

Sensaud de Lavaud, D. 1929. Vorrichtung zur Erzeugung eines Über- oder Unterdruckes in Gasen oder Flüssigkeiten. Deutsches Patentschrift Nr. 503775.

Tondu, B. 1997. Analysis and modeling of the dynamic behaviour of the McKibben artificial muscle. Reprints of the 5th IFAC Symposium on Robot Control, pp. 315-319, Nantes, France.

Tondu, B., Boitier, V. and Lopez, P. 1995. Théorie d'un muscle artificiel pneumatique et application à la modelisation du muscle artificiel de McKibben. Comptes Rendus de l'Académie des Sciences, Tome 320, Série IIb, pp. 105-114, Académie des Sciences, Paris.

Verrelst, B., Daerden, F., Lefeber, D., Van Ham, R. and Fabri, T. 2000. Introducing pleated pneumatic artificial muscles for the actuation of legged robots: a one-dimensional setup. Proceedings of CLAWAR 2000: Third International Conference, pp. 583-590, Madrid.

Winters, J. M. 1995. Braided artificial muscles: mechanical properties and future uses in prosthetics/orthotics. RESNA 13th Annual Conference, pp. 173-174, Washington DC. 


\section{Frank Daerden (1966)}

Study of Mechanical Engineering at the Vrije Universiteit Brussel. PhD in Applied Sciences, Vrije Universiteit Brussel, 1999. Research and teaching assistant at the Vrije Universiteit Brussel, 1991-1999. Doctor-Assistant at the dept. of Mechanical Engineering, Vrije Universiteit Brussel since 1999.
Dirk Lefeber (1956)

Study of Civil Engineering at the Vrije Universiteit Brussel. PhD in Applied Sciences, Vrije Universiteit Brussel, 1986. Professor, chairman of the department of Mechanical Engineering, head of the Multibody Mechanics Research Group, Vrije Universiteit Brussel. 